\title{
Análisis de las narrativas sobre desempeño ocupacional de trabajadores en pesca comercial
}

\author{
Aránzazu Pacheco Martínez ${ }^{a}$, Ana Isabel Souto Gómez ${ }^{b}$, Miguel Ángel Talavera Valverdec, \\ Pedro Moruno Miralles ${ }^{\mathrm{d}}$ \\ ${ }^{a}$ Facultad de Ciencias de la Salud, A Coruña, España. \\ bSecretaria y Comité Redactor de Revista TOG, A Coruña, España.

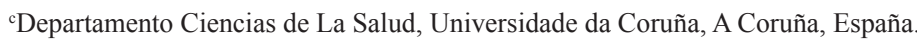 \\ ${ }^{d}$ Facultad de Terapia Ocupacional, Logopedia y Enfermería, Universidad de Castilla-La, Mancha, España.
}

\begin{abstract}
Resumen: La ocupación, entendida como aquella actividad con sentido en la que la persona participa cotidianamente y que puede ser nombrada por la cultura es lo que da sentido a nuestra vida. En la región Española de Galicia, las personas que trabajan en buques con actividad pesquera de altura, ven como la lejanía de sus hogares y las condiciones en las que tiene lugar su puesto de trabajo condicionan aspectos relacionados con su desempeño ocupacional y por consiguiente con su ocupación. Este artículo original de investigación tiene como objetivo principal conocer la vivencia del desempeño ocupacional de las personas que trabajan en una actividad productiva en alta mar. La metodología aplicada es de tipo cualitativa basándose en un estudio fenomenológico, enmarcado en un paradigma interpretativo. Se han utilizado como técnicas de recogida de datos, la entrevista en profundidad y el cuaderno de campo. El número total de informantes ha sido de 21 personas con edades comprendidas entre los 24 y 57 años. Los principales resultados de este estudio hacen referencia a las consecuencias derivadas del entorno en el desempeño ocupacional de los protagonistas. Ambos aspectos se encuentran presentes en los discursos, guardando relación con los aspectos extrínsecos e intrínsecos del trabajo desarrollado por los protagonistas del estudio. En cuanto a las conclusiones, se han identificado modificaciones en la gestión de la ocupación produciendo una repercusión tanto en la persona como la estructura familiar; todo ello motivado por la actividad productiva desarrollada.
\end{abstract}

Palabras clave: Terapia Ocupacional, Ocupación, Desempeño Ocupacional, Salud, Pesca Comercial.

\section{Análise de narrativas sobre o desempenho ocupacional dos trabalhadores na pesca comercial}

Resumo: A ocupação entendida como uma atividade com um sentido em que a pessoa participa diariamente e pode ser nomeado pela cultura é o que dá sentido à nossa vida. Na região espanhola da Galiza, as pessoas que trabalham em navios de pesca de altura, parecido com o afastamento de suas casas e as condições em que ocorre a condição de trabalho relacionadas com o seu desempenho profissional e, portanto, seus aspectos ocupação. O principal objetivo deste artigo original de pesquisa é conhecer a experiência do desempenho ocupacional das pessoas que trabalham em uma atividade produtiva offshore. A metodologia é baseada no tipo qualitativa um estudo fenomenológico, dentro de um paradigma interpretativo. Eles têm sido usados como técnicas de coleta de dados, entrevistas em profundidade e caderno de campo. O número total de entrevistados foi de 21 pessoas com idade entre 24 e 57 anos. Os principais resultados deste estudo referem-se às consequências da criação desempenho ocupacional dos protagonistas. Ambos os aspectos estão presentes em discursos, mas correlacionado com o trabalho extrínsecos e intrínsecos desenvolvido pelos protagonistas dos aspectos do estudo. Quanto aos resultados, identificamos mudanças na gestão da ocupação produzindo um impacto sobre o indivíduo e para a estrutura familiar; tudo motivado pela atividade produtiva desenvolvida.

Palavras-chave: Terapia Ocupacional, Ocupação, Desempenho Ocupacional, Saúde, Pesca Comercial.

Autor para la correspondencia: Aránzazu Pacheco Martínez, Dirección postal Calle Baiona, 3, 2izq, 36780 A, Guarda, Pontevedra, España, e-mail: zazu33389@hotmail.com.

Recibido Ene. 28, 2015; Revisión Mar. 23, 2015; Aceptado Mayo 04, 2015. 


\title{
Analysis of narratives about occupational performance of workers in commercial fishing
}

\begin{abstract}
Occupation, understood as an activity with a sense in which the person participates daily and can be named by the culture, is what gives meaning to our live. In the Spanish region of Galicia, people who work in deep-sea fishingfeel that the remoteness of their homes and the conditions under which occurs the workplace,could constrain aspects related to their occupational performance and therefore their occupation. This original research article's main aim is to know the experience of occupational performance of people working in a productive activity offshore. The methodology is a qualitative type, based on a phenomenological study, within an interpretive paradigm. They have been used as data collection techniques, in-depth interviews and field notebook. The total number of respondents was 21 people aged between 24 and 57 years old. The main results of this study refer to the consequences of environment in the occupational performance of the protagonists. Both aspects are present in speeches and related with extrinsic and intrinsic work developed by the protagonists of the study. As for the conclusions, we have identified changes in the management of the occupation producing an impact on both the individual and the family structure; all motivated by the productive activity developed.
\end{abstract}

Keywords: Occupational Therapy, Occupation, Occupational Performance, Health, Commercial Fishing.

\section{Introducción}

El desempeño ocupacional es motivo de estudio para los terapeutas ocupacionales. La Asociación Americana de Terapia Ocupacional (AOTA) lo define como la capacidad de hacer y completar una actividad o una ocupación seleccionada como resultado de una transacción dinámica entre la persona, el contexto/entorno, y la actividad (AMERICAN..., 2014).

Partiendo de este supuesto, un análisis del desempeño ocupacional permite conocer si las áreas, los patrones de desempeńo y los contextos o entornos pueden o podrían sufrir alguna modificación debido a factores extrínsecos o intrínsecos de la propia ocupación.

Por tanto, para la realización de este estudio serán analizados en profundidad conceptos relacionados con la ocupación humana tales como; desempeño ocupacional, contexto y entorno o patrones de desempeño (AMERICAN..., 2014).

\subsection{Importancia del tema}

En la comunidad autónoma donde se encuentra enmarcado este estudio, el desempeño ocupacional de la población del mismo, tiene unas características muy concretas debido a aspectos relacionados con el entorno en el que realizan su trabajo diario (CARRIL, 2006). Galicia es una comunidad autónoma española situada en el noroeste de dicho país y rodeada de mar en tres de sus cuatro provincias, por lo que mantiene una fuerte vinculación con la pesca en muchas de sus modalidades: extracción, conservación o comercialización (FUNDACIÓN..., 2012).

Partiendo de esta contextualización geográfica, social y cultural, la cual evidencia la fortaleza del vínculo con el sector pesquero, este estudio, se ha centrado en el análisis de las repercusiones que podrían surgir en el desempeño ocupacional de las personas que trabajan en buques con actividad pesquera de altura. Además, en él se estudia si dichos efectos, motivados por los constantes cambios de entorno, pueden afectar a la salud (LINCOLN; CONWAY, 1999).

\subsection{Antecedentes conceptuales}

El desempeńo ocupacional puede verse condicionado por diferentes aspectos de la ocupación tales como las funciones y estructuras corporales, los componentes psicológicos, las destrezas de ejecución, la demanda de la propia actividad a realizar o, por el entorno o contexto en el que ésta tenga lugar (NÚNEEZ, 2012).

El ser humano es un ser ocupacional (WILCOCK, 1998) y por ello, tiene la necesidad y capacidad de estructurar la ocupación para dar sentido la vida (SANZ; RUBIO, 2011). A la hora de estructurar la ocupación, se encuentran implícitos los procesos de adaptación y equilibrio de tal manera que se facilita el ajuste de las actividades cotidianas contribuyendo de esta forma al desarrollo de un exitoso desempeño ocupacional (NAVARRETE, 2010).

En este caso, uno de los principales factores que influyen en el proceso de adaptación es el entorno (GÓMEZ LILLO, 2006). La ocupación 
(ÁLVAREZ et al., 2007), no puede entenderse sin un entorno y un contexto, ni aislados de ellos. Concretamente en este estudio, el contexto y el entorno, son condicionantes de los restantes componentes del desempeńo ocupacional, en tanto que la ocupación de los implicados, depende lugar donde se desarrolla. Esto, según Hervás et al. (2014) genera situaciones de riesgo para la salud, tales como la disminución de la fuerza, el aumento del tiempo de reacción (LINCOLN; LUCAS, 2010a), la coordinación, la dificultad en tomar decisions (LINCOLN; LUCAS, 2010b), la pérdida de control sobre el equilibrio (ORGANIZACIÓN..., 2001a), alteraciones del estado de ánimo (LUCAS; LINCOLN, 2007), problemas de la salud mental, aumento de enfermedades crónicas o la muerte prematura según el Instituto Nacional de Seguridad e Higiene en el Trabajo - INSHT (INSTITUTO..., 2013), entre otras. Todo ello, tiene lugar en un entorno y contextos cambiante (característica principal de este tipo de trabajo) generando una modificación y compromiso del desempeńo ocupacional de la persona (RODRÍGUEZ; SUÂREZ DE VIVERO, 2003).

Por otro lado, en este estudio la salud abordada desde un enfoque biopsicosocial (ORGANIZACIÓN..., 2001b), proporcionando una visión desde una perspectiva tanto biológica, individual y social, teniendo en cuenta los factores ambientales que pueden ejercer un efecto en todos los componentes del funcionamiento (MORUNO; TALAVERA, 2011). Además, la condición de salud es un recurso para la vida diaria, para desempeñar la ocupación de una manera satisfactoria y disfrutar de ella, brindándonos diferentes oportunidades. Es por tanto un estado de bienestar físico, mental y social, no solo ausencia de enfermedad (MORUNO; TALAVERA, 2011).

\subsection{Definición del problema}

Dado que el principal objetivo de esta investigación son los efectos en la salud que puede conllevar este tipo de oficio, se profundiza en aspectos tales como el proceso de adaptación debido a los cambios en el desempeño ocupacional, los problemas derivados de las características del trabajo, un análisis de la salud, bienestar y calidad de vida, haciendo un especial hincapié en la influencia del entorno (AMERICAN..., 2014).

Principalmente, aspectos tales como las relaciones sociales, o las actividades de la vida diaria, se desarrollan dentro del buque. Por tanto, el buque es entendido, además de como vivienda, como el lugar donde se desarrollan actividades relacionadas con la participación social, relaciones de trabajo, entre otras
(RODRÍGUEZ; SUÁREZ DE VIVERO, 2003). El cuanto al buque como vivienda, debido a cuestiones de productividad en el aprovechamiento de espacio, abundan las zonas reducidas e incómodas, ya que los diseños de habitabilidad suelen ser bastante pobres en beneficio de los de almacenaje (EUROPEAN..., 2013). El buque de pesca, a diferencia de otros como los de la marina militar, se sitúa, en el extremo final del diseño de la habitabilidad. Estos aspectos estructurales, condicionan a otros como son la falta de intimidad o de espacio individual, que unidos a la sobrecarga de trabajo y la larga permanencia en la mar, generan un fuerte desarraigo social en los trabajadores. Además, Rodríguez y Suárez de Vivero (2003), concluyen en su estudio que esta situación empeora si la tripulación es heterogénea, es decir, formada por más de un grupo étnico; en cuyo caso la poca comunicación se limita a la actividad extractiva, impidiendo o dificultando la formación de un grupo y una adecuada convivencia.

Por todo ello, este trabajo de investigación pretende dar respuesta a la pregunta de investigación ¿̨cómo construyen el constructo vivencial del desempeño ocupacional las personas que trabajan en buques con actividad pesquera de altura?

\subsection{Objetivos}

De esta manera, el objetivo principal que subyace de este trabajo de investigación es el de conocer la vivencia del desempeño ocupacional de las personas que trabajan en una actividad productiva en alta mar.

Así mismo, existen otros objetivos secundarios, en los que también nos centraremos, siendo estos:

- Explorar las características básicas del desempeño ocupacional de los trabajadores con actividad productiva en alta mar.

- Indagar en la relación entre el desempeño ocupacional y la salud de los protagonistas.

- Identificar los elementos intervinientes en el equilibrio ocupacional de los trabajadores.

Como premisa de nuestra investigación, existe una hipótesis de estudio que es resultado de todas las teorías y supuestos relacionados con los objetivos de estudio. Por tanto, en este trabajo de investigación partimos de la hipótesis de que un cambio de entorno provoca una alteración en los patrones de desempeño; hábitos, rutinas y roles. Además este cambio de entorno puede tener efectos en la gestión de la ocupación y repercusiones en la salud. 


\section{Material y método}

\subsection{Diseño}

Este trabajo de investigación ${ }^{1}$ se encuadró dentro de una metodología cualitativa, aproximándose al fenómeno de estudio desde la vivencia de los informantes; con el fin de investigar la experiencia coetánea y el significado aportado a éstas. Esta aproximación permitió relacionar estos dos factores con el análisis de la salud subjetiva de cada persona.

Además se realizó una triangulación de informantes, con el objetivo de enriquecer el objeto de estudio con la información dada por cada uno de las personas o categorías de informantes. Los aspectos de rigor tenidos en cuenta con el propósito de aportar calidad al estudio han sido: credibilidad, confirmabilidad, transferibilidad y dependencia (HERNÁNDEZ et al., 2010).

\subsection{Población}

El estudio tuvo una duración de 9 meses, iniciándose en el 1 octubre de 2013y finalizandoel30 junio del 2014. Los procesos que se han seguido han sido, grosso modo, los de: revisión de la literatura, redacción de documentos relacionados con la ética de investigación, entrada al campo donde se realizaron entrevistas piloto, presentacióndel estudio a los participantes a través de los organismos colaboradores, recogida de datos a través de entrevistas, transcripción y análisis de datos, y por último, la redacción de los mismos.

La selección de informantes se llevó a cabo mediante un muestreo teórico intencionado abierto (FLICK, 2007), en el cual se han tenido en cuenta determinados criterios de selección bajo los cuales se condicionó la participación en el estudio. El principal organismo relacionado con la selección de informantes fue una cooperativa de pesca del Puerto de Vigo ${ }^{2}$ denominada ARVI (cuyas siglas pertenecen a Cooperativa de Armadores de Pesca del Puerto de Vigo.) Esta cooperativa está formada por 12 organismos de pesca, y de ellos, se ha seleccionado a una de las asociaciones teniendo en cuenta los criterios de mayor duración de los trabajadores en alta mar, y la representación de sus en aguas de diferentes zonas. Esta selección concluyó en la Asociación Nacional de Armadores de Buques de Palangreros de Altura (ANAPA).

A la hora de realizar la triangulación de informantes, con el objetivo de enriquecer el objeto de estudio con la información dada por cada uno de las personas o categorías de informantes, se han incluido tres categorías de informantes.
La muestra tuvo un número final de 21 informantes, con edades comprendidas entre los 24 y los 57 años. El lugar de residencia de estos informantes se situó en la ciudad de Vigo.

Cabe destacar que desde el primer contacto hasta la realización de la entrevista, no ha producido el abandono de ninguno de los participantes.

\subsection{Entorno}

El estudio se llevó a cabo en la ciudad de Vigo. Dicha ciudad se encuentra fuertemente vinculada con el sector pesquero debido principalmente a la importancia a nivel mundial de su puerto pesquero (SOUTELO, 2006). Por tanto, el enclave territorial elegido para este trabajo de investigación ha sido el puerto pesquero de la ciudad de Vigo denominado "O Berbés", situado en la parte occidental de la provincia de Pontevedra, integrada como municipio costero de las Rías Baixas.

Desde las épocas más antiguas hasta la actualidad, Galicia representa un referente en el sector de la pesca, ya que sus 123 puertos la convierten en la pesquería más importante de España y de Europa (SOUTELO, 2006). Además, la flota pesquera de Vigo se conforma como la más importante de Europa en capturas para el consumo humano, con un total de 742.204 toneladas de pescado. De esta manera, Vigo contribuye de manera fundamental a que Galicia sea la primera potencia pesquera de Europa. Por todo ello, la pesca es una señal de identidad de esta ciudad.

Además, el puerto de Vigo supone para la ciudad el escenario de la mitad de la actividad económica. El sector de la pesca extractiva tiene una gran importancia socioeconómica en esta ciudad debido a que emplea de manera directa a un total de 8.957 personas y teniendo en cuenta los empleos indirectos la población dependiente de este sector asciende a 32.679 personas (SOUTELO, 2006). Estas cifras, lo sitúan como el segundo sector productivo de Vigo, después de la automoción. Todo ello, representa el 40\% del Producto Interior Bruto (a partir de ahora será nombrado bajo las siglas PIB).

\subsection{Procedimiento}

La principal herramienta empleada para la recogida de información ha sido la entrevista en profundidad (OLABUÉNAGA, 2007) acompañada de la información recogida por el cuaderno de campo.

Este tipo de entrevista, permitió una amplitud y profundidad de contenido, y por otro que exige 
ausencia de dirección, concediendo de esta manera a los informantes extenderse en la temática planteada y comprender su perspectiva (OLABUÉNAGA, 2007). El eje temático de la entrevista se formuló en base a la vivencia ocupacional de las personas embarcadas y como a estas construyen su cotidianidad en base al contexto y al entorno donde desarrollen su actividad.

\subsection{Análisis de los datos}

El análisis de datos se realizó teniendo en cuenta la estructura teórica aportada en el texto Occupational therapy practice framework: Domain and process 3 nd $e d$. De esta manera, se analizaron los resultados de las grabaciones en formato audio con su posterior transcripción así como las anotaciones resultantes del cuaderno de campo. Estos resultados se presentaron con su respectivo código alfanumérico. Éste, se seleccionó teniendo en cuenta tres aspectos: por un lado el número de participante, en segundo lugar, su edad, y por último el tiempo (en años) que la persona lleva realizando la actividad productiva (embarcada). La privacidad y el anonimato de los informantes se respetaron mediante estos códigos alfanuméricos, a los cuales solo tuvieron acceso los investigadores. La recogida de datos se dio por finalizada una vez que se alcanzó la saturación teóricaes decir, en el momento en el que la selección de nuevos casos no produce información nueva o adicional. Los datos fueron transcritos en formato papel y la información resultante fue analizada e interpretada con el objetivo de recoger, analizar y sintetizar la información relevante para el estudio.

\section{Resultados}

Los principales resultados del estudio de investigación obtenidos se estructuraron en base a las categorías emergentes del análisis detallado de la información. Se presentaronclasificados en dos bloques con el objetivo de separar el tiempo en mar y en tierra, con dos secciones cada uno. En cuanto a las secciones, en la primera se tuvieron cuenta las áreas de ocupación y con ellas todos los aspectos que influyen para que se desarrollen. Estas han sido: actividades de alimentación, preparación de la comida, bañarse y ducharse, gestión de la comunicación, gestión y mantenimiento de la salud, descanso y sueño, educación, trabajo, y por último, ocio y tiempo libre.

En la segunda sección se abordaron los aspectos relacionados a los entornos y contextos en los que la ocupación tiene lugar. Estos han sido: entorno físico y social y contextos cultural, personal, temporal y virtual.

El hallazgo principal de este estudio en cuanto a los resultados del mismo está relacionado con la similitud de los testimonios en cuanto a la percepción de su desempeńo ocupacional. De esta manera, los resultados hacen referencia a todas las actividades que la persona realiza en su día a día, así como a aspectos relación al trabajo, el descanso y sueño o el ocio y tiempo libre.

A continuación se expresan mediante verbatims extraídos de las declaraciones de los protagonistas algunos ejemplos de resultados. Del periodo en el que los participantes se encuentran en el mar destacan las siguientes categorías:

En las actividades de la alimentación surgieron aspectos que tienen que ver con los patrones de desempeño, ya que el trabajo de la pesca se paraliza en función de esta actividad, que se realiza todos los días a la misma hora. Por otro lado, la rutina relacionada con esta actividad se modifica debido a eventos relacionados con el entorno tales como las circunstancias climatológicas adversas, afectando tanto a la asistencia de la propia actividad, como al modo en que se realice la misma. En sus discursos se observó la importancia y el valor que le dan al momento de la comida, incluso afirman que esta actividad llega a influir sobre otros aspectos del día a día.

Por relevos... a media mañana vas relevando a todo el mundo como si fuera una rueda, y comen un bocadillo uno, luego va otro... Y cuando es después al medio día, entonces van a comer la mitad de la tripulación, y la otra mitad continua levantando el aparejo (P.01 A.56 E.38).

En el caso de las actividades relacionadas con bañarse y ducharse, en relación a los patrones de desempeño afirmaron que esta actividad se realiza de manera adecuada en la actualidad, además de haber mejorado mucho en los últimos años.

Tenemos muy buenas condiciones, eres autosuficiente, tenemos potabilizadores y somos capaces de potabilizar unas 5-6 toneladas de agua al dia [...] somos 18 abordo y si te quieres duchar 3 veces al dia puedes, si hay 18 coladas se pueden hacer 18 coladas (P.02 A.46 E.30).

Las actividades vinculadas a la gestión de la comunicación, tienen como característica principal el hecho de no estar bajo el control de los protagonistas, los cuales dicen sentirse incomunicados y desconectados. Las demandas de la actividad 
requieren una conexión telefónica vía satélite, la cual supone un elevado coste.

En las actividades de gestión y mantenimiento de la salud, la mayor consecuencia se observa en la ausencia de disponibilidad de recursos. En cuanto a las características del cliente, en general prefieren no pensar en ello, ya que debido al entorno y a las circunstancias en las que están, hay factores como la disponibilidad inmediata de un servicio médico que son imposibles.

Mejor no pensar, porque si piensas no vas. Porque dices tú... si me pasa algo hospital no hay. Tardo 5 días o 8 en llegar... y o los que están aqui saben o si no...olvídate. Por eso que si piensas en eso malo...mejor no pensar (P.14 A.24 E.2).

La cantidad del sueńo en el periodo en el que los protagonistas de estudio están embarcados, en términos generales disminuye ya que la mayoría dicen necesitar menos horas para dormir con respecto al periodo de tierra. Sin embargo, aunque la cantidad de horas de descanso disminuya, la mayoría describen su descanso como "bueno" debido al cansancio acumulado de la jornada de trabajo.

El entorno social provoca una diferenciación en el desarrollo de los roles en el buque, que está condicionado al entorno. Los roles a los que hacen referencia la mayoría es a el de compañeros, y en general se consideran como una familia, apareciendo esta palabra en muchos de sus narraciones.

El barco es una casa, fuera de la parte del comedor $y$ los camarotes es un trabajo, es una casa y hay que acostumbrarse a ella. Y tu familia son ellos (P.08 A.44 E.28).

Del periodo en el que los participantes se encuentran en tierra destacan las siguientes categorías:

En las actividades derivadas de la gestión de la comunicación, los protagonistas reconocieron sentirse incomunicados y desconectados cuando llegan a tierra sobre las noticias o sucesos de actualidad que acontecieron mientras estaban ausentes.

Llegas como un emigrante o con el que estuvo en otro lado, con en el agravante que tuviste muy poca comunicación con la familia (P.21 A.58 E.30).

Los patrones de desempeño relacionados con las actividades de descanso y sueño, presentan como denominador común la dificultad para conciliar el sueño. Muchos aseguraron tener sueños con asuntos del barco, siendo esto más prevalente en informantes con cargos a bordo.
En casa cuesta el dormir seguido de un tirón... es raro... yo creo que nos pasa a casi todos que tenemos responsabilidades en los barcos... es dificil... porque siempre estas pendiente... estás en guardia permanente... [...] entonces eso sí que se trae a casa...si (P.02 A.46 E.30).

En cuanto a las actividades que implican participación social, si estando en el mar se aquejaban de estar "rodeados siempre de la misma gente", cuando llegan a tierra, el espectro de opciones se vuelve más amplio y esto les produce satisfacción. Además, este hecho provoca que surjan y se ańadan roles tales como el de amigo, tío o padre entre otros. La mayoría de ellos, refieren que el tiempo se les hace corto, y que quieren hacer muchas cosas.

Estresante, (risas) quieres hacer muchas cosas en poco tiempo. Normalmente es eso, quieres hacer de todo y...claro los días son los que son [...] (P.03 A.38 E.10).

\section{Discusión}

Ante la pregunta de investigación ¡cómo construyen el constructo vivencial del desempeño ocupacional las personas que trabajan en buques con actividad pesquera de altura?, este trabajo aporta evidencia sobre como es este constructo.

El desempeño ocupacional no es un proceso dinámico, así viene reflejado en su representación, entre la persona, el medio y las ocupaciones. La persona está conectada con el medio, de esta interacción nace la ocupación (VALDERRAMA; LARA, 2013). En este caso no podemos pasar por alto que al hablar de desempeño ocupacional, nos referimos a la capacidad de la persona de elegir, organizar y desarrollar de forma satisfactoria actividades significativas y culturalmente adaptadas, en búsqueda del cuidado personal y del disfrute de la vida, para contribuir a la sociedad (SIMO; URBANOWSKI, 2006). En este caso las cuestiones sociales presentes en esta investigación se ven claramente influidas por el desempeño ocupacional de los participantes. Por tanto podemos refutar que Galicia es una comunidad muy vinculada al sector pesquero, y esto se ve reflejado en los discursos de los protagonistas de esta investigación. Ya que a pesar de los factores que emergen de las situaciones de precariedad, u horarios interminables, o la lejanía del hogar, muy pocos de ellos abandonarían este trabajo si tuvieran la ocasión de hacerlo. Por tanto, podemos hablar de una "cultura del mar" que rodea y mantiene unidos a todos sus componentes y a sus familias generando cambios en los sistemas de 
valores socials (WHALEY; IWAMA, 2012) que redunda en la sociedad, o en los sistemas de salud, entre otros (POGGIE; POLLNAC, 2008).

Por todo ello y siguiendo las recomendaciones teóricas de Occupational therapy practice framework: Domain and process 3 nd ed., en la discusión de estos resultados, en relación a los conceptos introducidos desvelan que: por un lado, el significado que adquiere socialmente el trabajo en el mar en la comunidad gallega forma parte de la cultura de las personas, e influye sobre el desempeńo del mismo. La interpretación de los resultados y los hallazgos en la literatura, muestran como existe una modificación del desempeńo ocupacional de las personas que están embarcadas durante periodos de entre 2-4 meses, como es el caso de los protagonistas de este estudio.

Pueden ser múltiples causas las que influyen en esta situación, pero en este caso, entre otras cosas, es debido a: las condiciones que experimentan estando embarcados y los diferentes factores que influyen en el desarrollo del trabajo. De la misma manera, los protagonistas viven de manera muy diferente (POLLNAC; BAVINCK; MONNEREAU, 2012) su desempeño ocupacional estando a bordo y en tierra. Los resultados muestran como muchas de las actividades que se realizan a bordo, están influidas por diferentes aspectos implícitos del trabajo en un buque de altura. Los aspectos más relevantes, se pueden resumir en el buque como centro de trabajo y vivienda, la consecuente lejanía del hogar, el mar como entorno principal de desempeño ocupacional, las condiciones climatológicas en las que desarrollan la ocupación de los protagonistas y las tareas de pesca como eje en torno al cual gira y se desarrolla el día a día.

Por otro lado, se encuentran las consecuencias derivadas del entorno (AMERICAN..., 2014). En este entorno (que cuenta con peculiaridades tales como medio físico en movimiento, espacios reducidos, jornadas prolongadas y ritmo de trabajo intenso y elevado, entre otros) y haciendo referencia a los resultados obtenidos ocurren todos los aspectos de la ocupación, es decir el hombre, según Rodríguez y Suárez de Vivero (2003) "vive, trabaja y muere en su interior", pero también las convierte en lugar de ocio y recreo, aunque en este estudio los informantes no hacen especial hincapié en este área convirtiéndolo en un tiempo poco satisfactorio, si lo comparan con el tiempo que pasan en tierra (NGUYEN; LEUNG, 2013). El entorno físico en un buque se encuentra condicionado por aspectos de productividad y rendimiento, no por su habitabilidad (RODRIGUEZ; SUÁREZ DE VIVERO, 2003). Esto tiene unas repercusiones directas en la forma de mantener el desempeño ocupacional ya que los espacios son muy limitados y existe una exposición continua a factores de riesgo potenciales. Además, la persona no es protagonista ni tiene autonomía a la hora de adaptar el entorno a sus condiciones, deseos y voluntades (MORUNO; FERNÁNDEZ, 2012). Es por esto, que el entorno hostil en el que se desarrolla la ocupación de los protagonistas (debido principalmente a sus limitaciones y a los espacios restringidos), genera consecuencias en la competencia y equilibrio ocupacional (GÓMEZ LILLO, 2006) teniendo repercusiones en su desempeño ocupacional. Un ejemplo lo constituye la poca satisfacción de los protagonistas en el disfrute de su ocio y tiempo libre. Por otro lado, en este entorno nada favorecedor, para el desempeńo ocupacional, las actividades de la vida diaria instrumental, relacionadas con la gestión y mantenimiento de la salud, se ven modificadas en su ejecución, ya que entre otras, las demandas de la actividad cambian con respecto a tierra. Es decir, la manera de tener un control sobre aspectos de la salud, dependen de una tercera persona, que de manera telemática presta sus servicios de salud por medio de la radio del buque.

En cuanto a la relación con el estado de salud (VALDERRAMA; LARA, 2013), los resultados demuestran que este tipo de trabajo cuente con un alto índice de riesgo y peligrosidad (diferenciándolo del resto de trabajos) (BOND et al., 2004; JERRY, 2010), redundando en desempeño ocupacional y por consiguiente en la salud del protagonista (MORUNO; TALAVERA, 2011). La legislación Española al respecto, intenta controlar esta situación para evitar que eso suceda y es por ello que en el Art.15 de la Ley de prevención de Riesgos Laborales, 31/1995 de 8 de noviembre de 1995 (ESPAÑA, 1995), se establece la necesidad de:

[...] adaptar el trabajo a la persona, en particular en lo que respecta a la concepción de los puestos de trabajo, así como a la elección de los equipos y los métodos de trabajo y de producción, con miras, en particular a atenuar el trabajo monótono y repetitivo y a reducir los efectos del mismo en la salud.

Además, el trabajo de la pesca de altura ha sufrido grandes avances y mejoras en el periodo de los últimos 20-30 años y aunque la aparición de las nuevas tecnologías a bordo, ha generado una retracción de la socialización, la participación y las relaciones, el avance a resultado prometedor y meteórico en legislación (tal y como corroboran los resultados de este estudio). En primer lugar, en la actualidad la legislación, que es más sólida en materia de derechos fundamentales a bordo y calidad de vida. En segundo lugar, el devenir de 
las mejoras en el transcurso de los ańos dedicados a esta actividad, ha generado un ajuste interno en cada empresa en estos aspectos, primando y dando valor a las comodidades de los trabajadores.

Un ejemplo de ello lo constituye la incorporación de potabilizadoras a bordo. Este avance ha propiciado un cambio en las actividades de aseo, como consecuencia de un cambio de patrones de desempeńo relacionado con el proceso de transformación e innovación de los buques, en tanto que en la actualidad, todos los buques llevan potabilizadoras de agua, las cuales permiten un uso ilimitado de la misma. Por tanto, una actividad que antaño era una excepción, se ha convertido en algo rutinario y normalizado que se ha automatizado, generando un hábito.

\subsection{Implicación para la práctica}

Este estudio presenta una aplicabilidad tanto investigadora como de comunidad. En el primer caso, el estudio contribuye a la obtención de nuevos datos y líneas de investigación sobre la relación del desempeño ocupacional y la pesca comercial, pudiendo arrojar nuevas pistas sobre futuras investigaciones.

En cuando a la aplicabilidad en la comunidad, los resultados y conclusiones de este estudio, ofrecen un conocimiento que se va a extraer sobre el desempeño ocupacional y la ocupación de las personas que trabajan en la mar. Esto permite realizar un análisis de esta situación, usando para ello la incorporación de recomendaciones que aporta la literatura científica de otros países y de esta manera aplicar los conocimientos a la realidad de nuestra comunidad autónoma.

\subsection{Recomendaciones para futuras investigaciones}

De esta manera, las recomendaciones y sugerencias para investigaciones futuras que podemos recomendar van encaminadas a una comparación entre los diversos tipos de pesca, y cómo algunas artes, tradiciones o condiciones laborales facilitan un mejor desempeño ocupacional, conciliación familiar y mejores condiciones de trabajo. Un ejemplo podría ser, la comparación con las condiciones de los buques vascos atuneros que cuentan con jornadas de trabajo más estructuradas y más similares a las de un trabajo en tierra.

\subsection{Limitaciones del estudio}

Los errores metodológicos que existen a lo largo del proceso de investigación son los siguientes: en primer lugar los relacionados con la selección de informantes, ya que el acceso a las personas que trabajan en buques con actividad pesquera de altura, se velimitado por los cortos periodos vacacionales, momento en el cual serealizan las entrevistas. Además, podemos destacar la limitación de la universalización de las conclusiones, la cual se ve limitada por la metodología utilizada, sin embargo no es una pretensión de esta investigación la universalización sino que se puedan transferir los resultados a contextos similares.

\section{Conclusiones}

Para finalizar este trabajo de investigación nos gustaría puntualizar las conclusiones que se extraídas:

- El entorno donde se lleva a cabo una actividad productiva es influyente y determinante tanto para la persona como para su núcleo y estructura familiar; determinando las relaciones y la periodicidad de ellas.

- El entorno donde se desarrolla la actividad de los protagonistas, produce cambios en el desempeño ocupacional (hábitos y rutinas) y en la gestión de la ocupación, conllevando a un desequilibrio ocupacional que repercute en la salud de las personas que ejercen un trabajo en alta mar.

- La transición ocupacional que se produce cuando la persona modifica su entorno influye en la adaptación de los patrones de desempeño.

- Por todos los resultados obtenidos en este trabajo, nos aventuramos a asegurar que la intervención de Terapia Ocupacional en esta población tiene un papel relevante, asistiendo al proceso de reorganización del desempeño ocupacional y por consiguiente al ajuste de la condición de salud.

\section{Referencias}

ÁLVAREZ, E. et al. Definición y desarrollo del concepto de ocupación: ensayo sobre la experiencia de construcción teórica desde una identidad local. Revista Chilena de Terapia Ocupacional, Santiago de Chile, v. 7 , p. 76-82, 2007.

AMERICAN OCCUPATIONAL THERAPY ASSOCIATION - AOTA. Occupational therapy practice framework: domain and process. American Journal of Occupational Therapy, New York, v. 68, n. 8, p.1-47, 2014. Suplemento 1. 
BOND, C. et al. The health and lifestyle of Scottish fishermen: a need for health promotion. Health Education Research, Atlanta, v. 19, n. 4, p. 373-379, 2004.

CARRIL, X. M. Algunas de las especificidades más problemáticas del trabajo en el mar realizado a bordo de embarcaciones pesqueras. Revista Galega de Economía, Santiago de Compostela, v. 15, n. 1, p. 1-15, 2006.

ESPAÑA. Ley de prevención de Riesgos Laborales. Ley $31 / 1995$ de 8 de noviembre de 1995. Boletín Oficial del Estado, no 269, Madrid, 08 nov. 1995. p. 32590-32611.

ESPAÑA. Ley orgánica de Protección de Datos de Carácter Personal. Ley 15/1999 de 13 de diciembre 1999. Boletín Oficial del Estado, no 298, Madrid, 13 dic. 1999. p. 43088-43099.

ESPAÑA. Ley reguladora del consentimiento informado y de la historia clínica de los pacientes. Ley 3/2001 de 28 de mayo de 2001. Boletín oficial del Estado, no158, Madrid, 28 mayo 2001. 9 p.

ESPAÑA. Ley reguladora del consentimiento informado y de la historia clínica de los pacientes Ley 3/2005, de modificación de la Ley 3/2001, de 28 de mayo de 2005. Boletín Oficial del Estado, no 93, Madrid, 28 mayo 2005. p. 13364-13368.

EUROPEAN COMMISSION. Un nuevo acuerdo de los interlocutores sociales prepara el camino para mejorar las condiciones laborales de la pesca. Bruselas. Disponible: <http://europa.eu/rapid/press-release_IP-12-493_ es.htm>. Acceso: 17 dic. 2013.

FLICK, U. Introducción a la investigación cualitativa. A Coruña: Fundación Paideia Galiza, 2007.

FUNDACIÓN PARA LA PESCA Y EL MARISQUEO - FUNDAMAR. Análisis de la aplicación de la PRL en el sector de la pesca en Galicia: propuesta de intervención. Vigo. Xunta de Galicia, 2012.

GÓMEZ LILLO, S. Equilibrio y organización de la rutina diaria. Revista Chilena de Terapia Ocupacional, Santiado de Chile, v. 6, p. 47-54, 2006.

HERNÁNDEZ, R.; FERNÁNDEZ, C.; BAPTISTA, M. P. Metodología de la investigación cualitativa. México: McGraw-Hill/Interamericana, 2010.

HERVÁS, P. et al. Sector pesquero: análisis de situación de la salud laboral. Madrid: Instituto Nacional de Seguridad e Higiene en el Trabajo, 2014.

INSTITUTO NACIONAL DE SEGURIDAD E HIGIENE EN EL TRABAJO -INSHT. Madrid. Disponible: <http://www.insht.es/portal/site/Insht/>. Acceso: 17 dic. 2013.

JERRY, M. E. The development and efficacy of safety training for commercial fishermen. Journal of Agromedicine, Marshfield, v. 15, n. 4, p. 1-6, 2010.

LINCOLN, J. M.; CONWAY, G. A. Preventing commercial fishing deaths in Alaska. Occupational Enviroment Medical, Melbourne, v. 56, p. 691-695, 1999.
LINCOLN, J. M.; LUCAS, D. L. Fatal occupational injuries in the U.S. Commercial Fishing Industry: risk factors and recommendations. Anchorage: National Institute for Occupational Safety and Health, 2010a.

LINCOLN, J. M.; LUCAS, D. L. Occupational fatalities in the United States commercial fishing industry, 2000-2009. Morbidity and Mortality Weekly Report, Atlanta, v. 59, n. 27, p. 842-845, 2010 b.

LUCAS, D. L.; LINCOLN, J. M. Fatal fallsoverboardon comercial fishing vessels in Alaska. American Journal of Industrial Medicine, New York, v. 50, n. 12, p. 962 968, 2007.

MORUNO, P.; FERNÁNDEZ, P. Análisis teórico de los conceptos privación, alienación y justicia ocupacional. TOG (A Coruña), Galícia, v. 9, n. 5, p. 44-68, 2012. Disponible: <www.revistatog.com/mono/num5/teorico. pdf>. Acceso: 25 oct. 2013

MORUNO, P.; TALAVERA, M. A. Ocupación y calidad de vida. In: MORUNO, P.; TALAVERA, M. A. Terapia Ocupacional en Salud Mental. Barcelona: Elsevier Masson, 2011. p. 21-36.

NAVARRETE, E. Ciencia de la Ocupación: caminos y perspectivas. TOG (A Coruña), Galícia, v. 7, n. 6, p. 132-143, 2010. Disponible: <www.revistatog.com/suple/num6/erna.pdf>. Acceso: 3 dic. 2013.

NGUYEN, Q.; LEUNG, P. Do Fishermen Have Different Attitudes Toward Risk? An Application of Prospect. Theory to the Study of Vietnamese Fishermen. The Australian Journal of Agricultural and Resource Economics, Malden, v. 34, n. 3, p. 518-538, 2013.

NÚNEEZ, A. Ocupación, cultura y sociedad: el entorno social y cultural como clave para el éxito de la Terapia Ocupacional. TOG (A Coruña), Galícia, v. 9, n. 5, p. 32-43, 2012. Disponible: <www.revistatog.com/mono/ num5/clave.pdf>. Acceso: 17 oct. 2013.

OLABUÉNAGA, J. I. R. Metodología de la Investigación cualitativa. Bilbao: Universidad de Deusto, 2007.

ORGANIZACIÓN MARÍTIMA INTERNACIONAL. Orientaciones acerca de la reducción y gestión de la fatiga. Londres: OMI, 2001a. (MSC/Circ.1014).

ORGANIZACIÓN MUNDIAL DE LA SALUD. Clasificación Internacional del funcionamiento, de la discapacidad y de la Salud: CIF. Madrid: Ministerio de Trabajo y Asuntos Sociales, 2001b.

POGGIE, J. J.; POLLNAC, R. B. Happiness, wellbeing and psychocultural adaptation to the stresses associated with marine fishing. Human ecology review Bar, Harbor, v. 15, n. 2, p. 194-200, 2008.

POLLNAC, R.; BAVINCK, M.; MONNEREAU, I. Job satisfaction in fisheries compared. Social Indicators Research, Boston, v. 109, p. 119-133, 2012.

RODRÍGUEZ, J. A.; SUÁREZ DE VIVERO, J. L. El buque como vivienda: una aproximación metodológica. Scripta Nova Revista Electrónica de Geografía y Ciencias 
Sociales, Barcelona, v. 7, n. 149 , p. 1-28, 2003. Disponible: <www.ub.es/geocrit/sn/sn-149.htm>. Acceso: 6 nov. 2013.

SANZ, P.; RUBIO, C. Ann Allart Wilcock: trayectoria profesional y aportaciones más relevantes a la terapia ocupacional y a la ciencia de la ocupación. TOG $(A$ Coruña), Galícia, v. 8, n. 14, p. 1-18, 2011. Disponible: $<$ www.revistatog.com/num14/pdfs/historia1.pdf $>$. Acceso: 5 sep. 2013.

SIMO, S.; URBANOWSKI, R. El modelo Canadiense del desempeño ocupacional I. TOG (A Coruña), Galícia, v. 3, n. 3, p. 1-27, 2006. Disponible: <http://www.revistatog.com/num3/pdfs/ExpertoI.pdf>. Acceso: 6 dic. 2013.
SOUTELO, C. M. L. El papel de los puertos en el desarrollo del sector pesquero: el puerto de Vigo. Revista Galega de Economía, Galícia, v. 15, n. 1, p. 1-8, 2006.

VALDERRAMA, C.; LARA, P. Cuestionamientos sobre el carácter beneficioso para la salud y el bienestar de la ocupación: la emergencia de la ocupación como fenómeno social. TOG (A Coruña), Galícia, v. 10, n. 18, p.1-15, 2013. Disponible: <www.revistatog.com/num18/pdfs/ original9.pdf $>$. Acceso: 3 oct. 2013.

WHALLEY, K. R.; IWAMA, M. Well-being and occupational rights: an imperative for critical occupational therapy. Scandinavian Journal of Occupational Therapy, Oslo, v. 19, p. 385-394, 2012.

WILCOCK, A. A. An occupational perspective of health. Thorofare: Slack, 1998.

\section{Contribución de los Autores}

Los autores firmantes hemos estado estrechamente involucrados en la concepción y diseño de la investigación, hemos asumido la responsabilidad de la recolección e interpretación de datos, redacción de la publicación, modificación del manuscrito tras los comentarios de los revisores por pares y hemos aprobado la versión final de del documento para que se ajuste a los criterios de la publicación.

\section{Notas}

${ }^{1}$ Como muestra de rigor metodológico se ha hecho entrega a todos los participantes hojas de presentación e información acerca del estudio. También se han seguido las recomendaciones legales en referencia al consentimiento informado, de tal manera ha sido indispensable para la participación el estudio, la firma del citado documento. Estos documentos han sido redactados teniendo en cuenta la Ley reguladora del consentimiento informado y de la historia clínica de los pacientes 3/2005 de 28 de mayo de 2005 (ESPAÑA, 2005), que modifica la Ley reguladora del consentimiento informado y de la historia clínica de los pacientes 3/2001 de 28 de mayo de 2001 (ESPAÑA, 2001), salvaguardándose la confidencialidad de la información amparándose en la Ley orgánica de Protección de Datos de Carácter Personal, 15/1999 de 13 de diciembre 1999 (ESPAÑA, 1999). Esta investigación se desarrolló bajo el cumplimiento de las normas del Convenio relativo a los derechos humanos y biomedicina del Consejo de Europa y el respeto a las normas de buena práctica clínica de la Declaración de Helsinki. Esta investigación tiene el informe favorable con el código (2013/539) del Comité Autonómico de Ética de la Investigación de Galicia.

2 Ciudad de Vigo, Pontevedra, Galicia, España. 